\title{
Thermal Properties between SAN and ABS Copolymer Matrices Blended with PTFE Polymer Particle Composites
}

\author{
R. Mahesh Kumar, N. Rajini
}

\begin{abstract}
Styrene Acrylonitrile (SAN) copolymer has styrene with high expanded durability and finds some useful applications. Applications involving replacement of metal by plastics due to light-weight, high quality and firmness attributes were seen. But they are limited as they cannot withstand higher temperature applications. PTFE that finds a perfect filler material has high temperature ability, self-greasing up, high chemical inertness, low friction, corrosion resistance, low outgassing properties. Injection molding process helped addition of copolymers of SAN and ABS to 10\% PTFE by weight, $20 \%$ PTFE by weight blends and composite samples were prepared successfully using the melt condition. Experimental results show PTFE filler added composites exhibiting improved thermal conductivities and coefficient of linear thermal expansion.
\end{abstract}

Keywords: Styrene Acrylonitrile (SAN) and Acrylonitrile Butadiene Styrene (ABS) matrices, Polytetrafluroethylene (PTFE) particle reinforcement,Comparison of Thermal properties and Injection Molding.

\section{INTRODUCTION}

Utilization of polymer composite-based materials is on the increase [1]. The constantly developing requirement for polymer composite materials brings about the formation of different polymer blends with various compositions, morphologies and properties [2]. Thermal analysis is essential for obtaining better product design and quality control over polymer composite products.

The SAN/PTFE and ABS/PTFE composite samples are manufactured by injection molding machine using plastic molds by heating to melt condition to ensure homogenous mix of PTFE particles. Polymer composites thermal properties also confirmed by examining them by various graphs obtained from the results of TGA.

Styrene-acrylonitrile copolymer has 20 to $30 \%$ acrylonitrile contentand Chemical Formula is $\left\{\left[\mathrm{C}_{3} \mathrm{H}_{3}-\mathrm{N}\right]_{\mathrm{n}}\right.$ $\left.\left[\mathrm{C}_{8} \mathrm{H}_{8}\right]_{\mathrm{n}}\right\}$ with increasing the content helping improvement to toughness and chemical resistance but finding difficulty in the moulding process. In general, the nature of the molecular structure has caused a tendency for SAN resin to be yellow and lose transparency after injection moulding.

Revised Manuscript Received on December 15, 2019.

* Correspondence Author

R. Mahesh Kumar*, Research Scholar, School of Automotive and Mechanical Engineering, Kalasalingam Academy of Research and Education, Krishnankoil, India.Email: maheshkumarb.e.mca@gmail.com

N. Rajini, School of Automotive and Mechanical Engineering, Kalasalingam Academy of Research and Education, Krishnankoil, India.

Email:rajiniklu@gmail.com
Acrylonitrile Butadiene Styrene (ABS) is an amorphous thermoplastic polymer transparent with the chemical formula $\left.\left(\mathrm{C}_{8} \mathrm{H}_{8}\right)_{\mathrm{x}} \cdot\left(\mathrm{C}_{4} \mathrm{H}_{6}\right)_{\mathrm{y}} \cdot\left(\mathrm{C}_{3} \mathrm{H}_{3} \mathrm{~N}\right)_{\mathrm{z}}\right)$. The proportions can shift from 40 to $60 \%$ styrene, 15 to $35 \%$ acrylonitrile and 5 to $30 \%$ butadiene. ABS has low melting point and facilitating the use in 3D printing, mannequins, toys, sanitary ware, auto components, sports equipment, and furniture.

Polytetrafluroethylene (PTFE) is a man made fluro polymer of tetrafluroethylene. The chemical formula for PTFE $\operatorname{isn}\left[\mathrm{F}_{2} \mathrm{C}=\mathrm{CF}_{2}\right] \rightarrow-\left[\mathrm{F}_{2} \mathrm{C}-\mathrm{CF}_{2}\right]_{\mathrm{n}}-$. The thermo plastic polymer, $\mathrm{PTFE}$ is white solid at room temperature. Processing procedure for PTFE is difficult, due to high melting temperature $\left(327^{\circ} \mathrm{C}\right)$. PTFE is best used in coating non-stick frying pans and other cookware. PTFE plays a vital role in the medical field such as surgical equipment and machinery.

\section{MATERIALS AND METHODS}

SAN and ABS polymer pellets were procured from SreeMeenakshi Plastics, Madurai, Tamilnadu, India. Powder PTFE polymer in powder procured from M/s. Modoplast Company Pvt. Ltd, Kolkata, West Bengal, India. Average diameter of SAN and ABS pellets were approximately $2 \mathrm{~mm}$. The particles sizes of PTFE powder were about 5 to $15 \mu \mathrm{m}$. Sample specimens size 200x140x $3 \mathrm{~mm}^{3}$ polymer mold made from EN8 mild steel plates were procured from Mahesh Plastics, Madurai, Tamilnadu, India.

Samples used in this research work were SAN-1: $100 \%$ SAN by weight, SAN-2: with SAN $+10 \%$ by weight composition of PTFE powder particle, SAN-3: with SAN + $20 \%$ by weight composition of PTFE powder particle, ABS1: $100 \%$ ABS by weight, ABS-2: with ABS $+10 \%$ by weight composition of PTFE powder particle and ABS-3: with $\mathrm{ABS}+20 \%$ by weight composition of PTFE powder particle [3]. Individually SAN pellets, ABS pellets and PTFE powder were pre-dried to moisture free condition at $100^{\circ} \mathrm{C}$ in vacuum oven for $24 \mathrm{~h}$. Materials as per requirement were taken in six batches with different weight composition, mentioned as SAN-1, SAN-2, SAN-3, ABS-1, ABS-2 and ABS-3 one by one fed into the hopper and molded test plate samples are made [4].

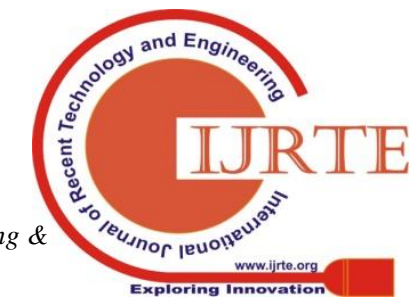




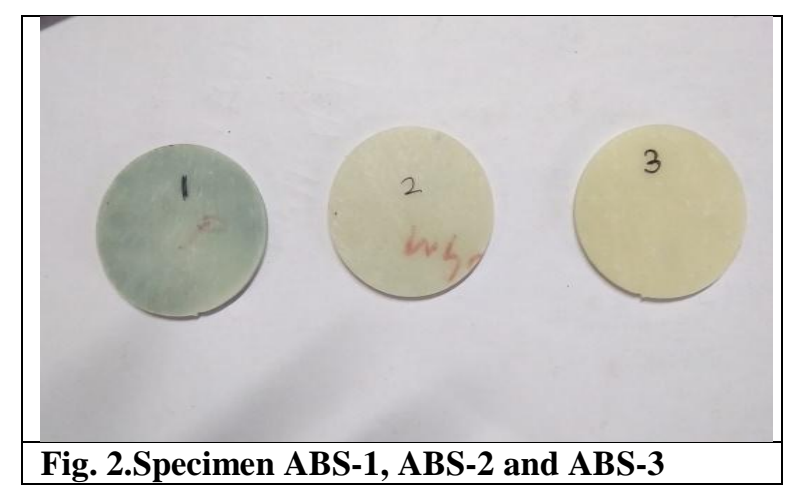

III. THERMAL PROPERTIES

\section{A. Thermal conductivity measurement}

Thermal conductivity measurements were conducted under thermal equilibrium conditions. A round shaped molded specimen with $50 \mathrm{~mm}$ diameter and $3 \mathrm{~mm}$ thickness as per ASTM E1530 were utilized for thermal conductivity estimates for all samples [5]. Steady state heat was applied from one side of the specimen until thermal equilibrium was achieved. The temperatures of the top and bottom surfaces were estimated with the help of thermocouples. The experiments were conducted in the temperature range of 25 to $90^{\circ} \mathrm{C}$.

\section{B. Coefficient of Linear Thermal Expansion (CLTE)}

The test specimens with size $90 \times 10 \times 3 \mathrm{~mm}$ were used for CLTE test. CLTE tests were done according to ASTM D696 at 25 to $90^{\circ} \mathrm{C}$ utilizing electrical heater. The specimens were heated under steady state from 30 to $90^{\circ} \mathrm{C}$ and equilibrium was maintained on $90^{\circ} \mathrm{C}$ for 10 minutes.

\section{Thermogravimetric Analysis (TGA)}

The thermal degradation behavior of composite samples was examined at King Mokut's University laboratory, Thailand using Mettler - STAR SW 15.00. Powdered samples $10 \mathrm{mg}$ were obtained by hacksawing the plate samples of polymer composites used for testing. With a change in mass of the sample as a function of temperature rate $10^{\circ} \mathrm{C} \mathrm{min}^{-1}$, TGA recorded the thermal degradation

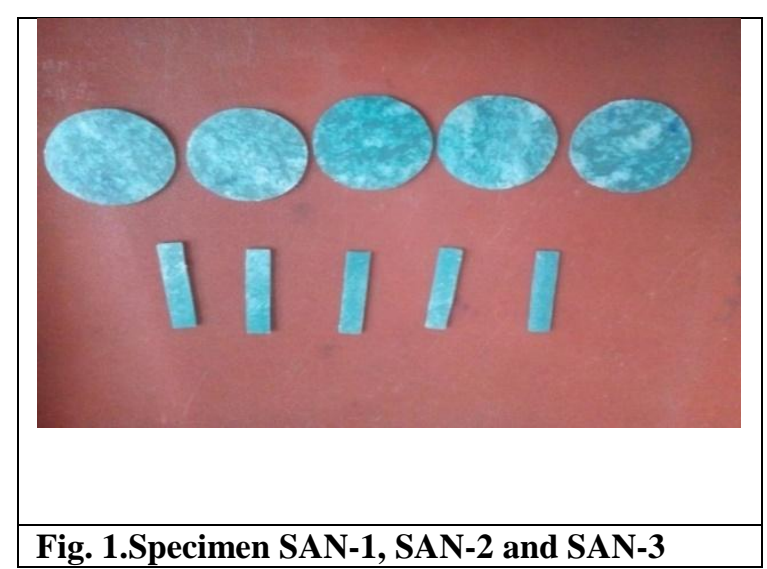

process [6]. The nitrogen gas $60 \mathrm{ml} \mathrm{min}^{-1}$ environment was selected for polymer composite sample thermal decomposition with temperature range from $30-700^{\circ} \mathrm{C}$.

\section{RESULTS AND DISCUSSION}

Comparison values for thermal conductivity and coefficient of linear thermal expansion for different samples are presented in bar chart. The TGA comparison curves are plotted and analyzed.

\section{A. Thermal Conductivity Test Report:}

The acquired outcomes showed a comparison of ABS copolymer having twice higher thermal conductivity than SAN copolymer [7]. The write up showed blended PTFE particles having effective thermal conductivity in the light of the fact that PTFE powder established thermal percolation threshold. The blended composites were filled by $10 \%$ by wt. PTFE and $20 \%$ by wt. PTFE exhibited a better thermal conductivity than base copolymers. Comparison analysis showed, SAN composites exhibiting high thermal conductivity than ABS composites. Thus addition of PTFE proves effective for SAN polymer composite and peak values $0.175 \mathrm{~W} / \mathrm{mK}$ and $0.162 \mathrm{~W} / \mathrm{mK}$ were recorded.

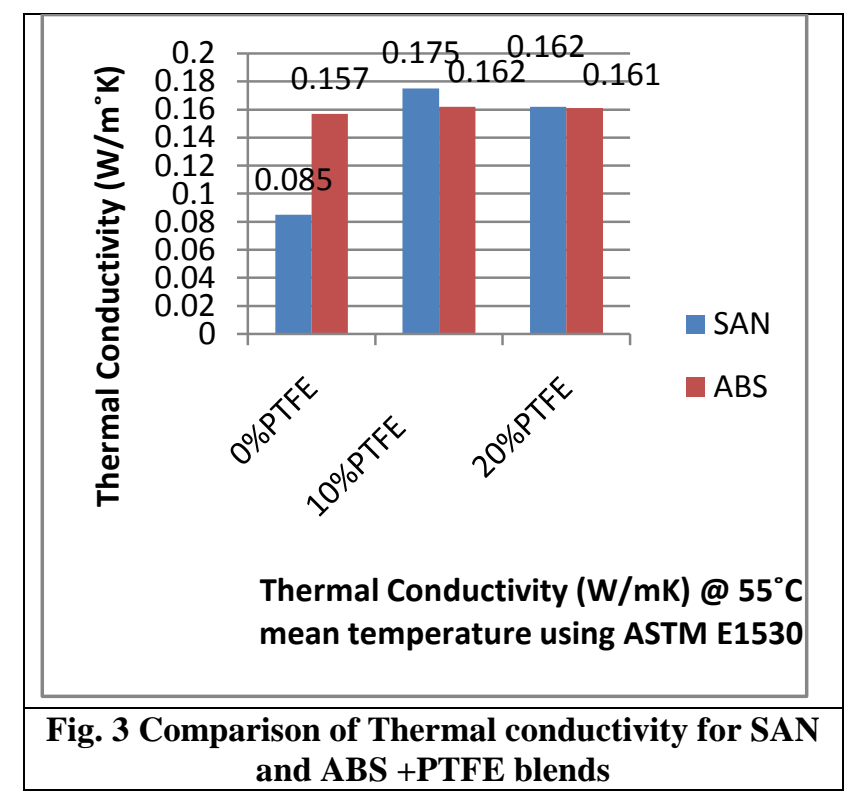

B. Coefficient of Linear Thermal Expansion Test Report

CLTE examination was done as per ASTM standard D696 between operating temperatures $30-60^{\circ} \mathrm{C}$ and values $/{ }^{\circ} \mathrm{C}$ were obtained for all samples. Outcomes of the investigation showed SAN copolymer having a low CLTE value compared with ABS copolymer. Similarly the addition of PTFE encouraged the coefficient of linear thermal expansion values. SAN polymer composites filled with $20 \%$ wt. PTFE showed an extraordinary high CLTE value $\left(6.66 \times 10^{-5}\right)$ compared to ABS composite of same $20 \%$ wt.

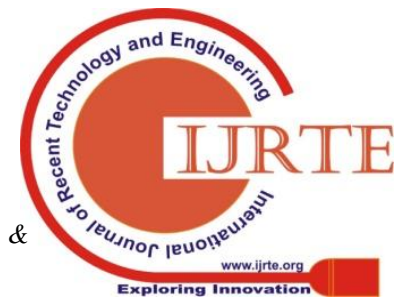


PTFE. The experimental estimations of the CLTE values of all samples are displayed in a bar chart.

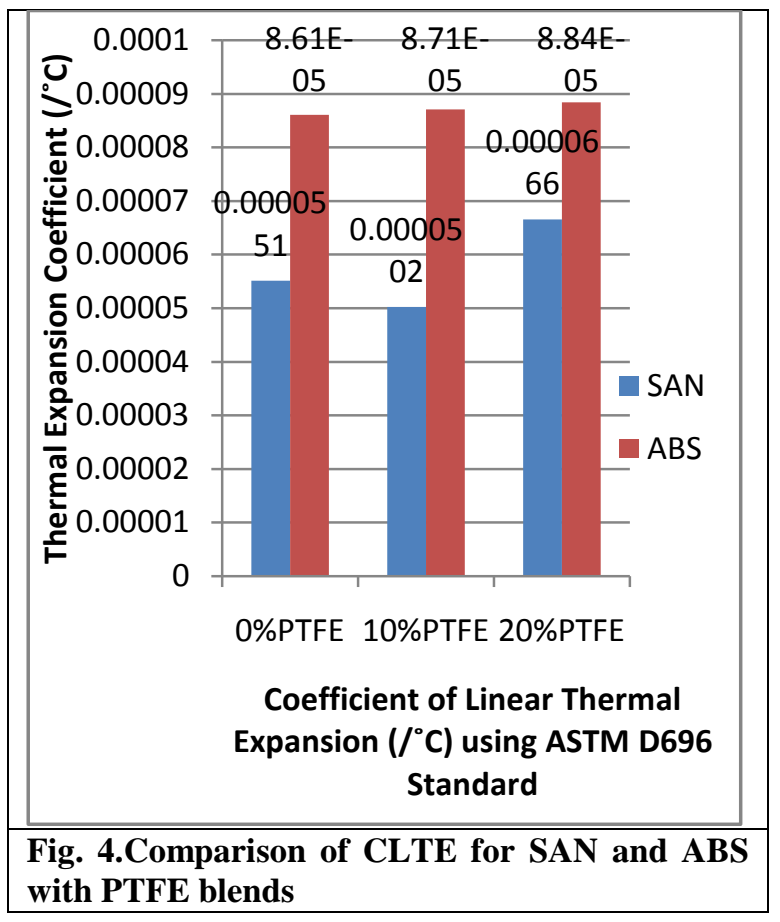

\section{TGA Test Report}

TGA provides quantitative estimation of mass change with time and temperature in polymer composite samples related to transition and thermal degradation [8]. The polybutadiene component of ABS is the weakest part of ABS. A reaction scheme showing some pathways of degradation is shown in Fig. 5. The initial radical came from thermal decomposition of another component in the sample. The results of comparison for SAN and ABS blends are given as graphs.

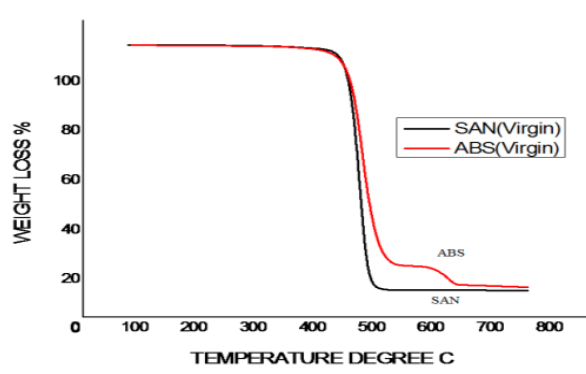

Fig. 5.Primary thermograms of SAN and ABS copolymers

The graph clearly shows an increase in the thermal stability of the copolymers with the addition of PTFE. Particles added $10 \%$ by wt. showed increase in thermal stability for ABS copolymer composites and particles added $20 \%$ by wt. showed increase in SAN copolymer composites. The high specific area of SAN+20\% PTFE provided better interaction between PTFE particle and SAN matrix that tends to increase in interfacial bonding. Also the char content of composites were higher than the copolymer matrix alone, considering higher thermal stability of PTFE particles than copolymer matrix.

\section{CONCLUSIONS}

The observations from the results show the display of most extreme thermal conductivity $(0.175 \mathrm{~W} / \mathrm{mK})$ and $20 \%$ by weight composition with $0.162 \mathrm{~W} / \mathrm{mK}$ by SAN polymer composited blended with $10 \%$ weight PTFE. The write up shows PTFE particles consuming more thermal conductivity stuff due to PTFE powder up grading the heat transfer rate [9]. ABS composites blended with (10\% Wt.) PTFE particles had good thermal conductivity $(0.162 \mathrm{~W} / \mathrm{mK})$. Moreover SAN/PTFE particles displayed good thermal properties while related with overall results determined.

\section{REFERENCES}

1. Anna G. Koniuszewska and Jacek W. Kaczmar, "Application of polymer based composite materials in transportation," Smithers information Ltd., vol. 32, 2016, 1.

2. YibingCai, Yuan Hu, Junfeng Xiao, Lei Song, Weicheng Fan, Huaxia Deng, Xinglong Gong and Zuyao Chen, Morphology, "Thermal and Mechanical Properties of Poly (Styrene-Acrylonitrile) (SAN) / Clay Nanocomposites from Organic-Modified Montmorillonite," PolymerPlastics Technology and Engineering, vol. 46, 2007, pp. 541-548.

3. A. Aruniit, J. Kers and K. Tall, "Influence of filler proportion on Mechanical and Physical properties of particulate composite," Agronomy Research Biosystem Engineering Special issue, vol. 1, 2011, pp. 23-29.

4. Manish Kumar Lila, SaurabhChaitanya, Inderdeep Singh, Faninder Kumar, Sanjay Sharma, "Mechanical behaviour of injection moulded bagasse fibre reinforced PP and PE composites, ResearchGate," Processing and Fabrication of Advanced Materials XXV, 2017.

5. S. Zhang, X. Y. Cao, Y. M. Ma, Y. C. Ke, J. K. Zhang, F. S. Wang, "The effects of particle size and content on the Thermal conductivity and Mechanical properties of $\mathrm{Al}_{2} \mathrm{O}_{3}$ /High density polyethylene (HDPE) composites," eXPRESS Polymer Letters, vol. 5, 2011, pp. 581-590.

6. V. Selvakumar, N. Manoharan, "Thermal properties of Polypropylene/Montmorillonitenanocompsites," International Journal of Science and Technology, vol. 7, 2014, pp. 136-139.

7. MadhusreeKole, D. Tripathi, T. K. Dey, "Percolation based enhancement in effective thermal conductivity of HDPE/LBSMO composites," Building material Science, vol. 35, 2011, pp. 601-609.

8. Carola Esposito Corcione and MariaenricaFrigione, "Characterization of Nanocomposites by Thermal analysis," Materials, vol. 5, 2012, pp. 2960-2980.

9. I. M. Inuwa, A. Hassan, S. A. Shamsudin, "Thermal properties, structure and morphology of Graphene reinforced Polyethylene Terephthalate / Polypropylene Nanocomposites," The Malaysian Journal of Analytical Sciences, vol. 18, 2014, pp. 466-477.

\section{AUTHORS PROFILE}

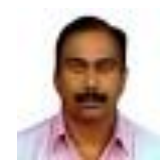

R. Mahesh Kumar, is currently Assistant Professor in the Department of Mechanical Engineering, NPR College of Engineering and Technology, Natham, India. He received his Ph.D. degree in Kalasalingam Academy of Research and Education, Krishnankoil, India. His main area of research interest is thermal analysis on polymer composites. 
Thermal Properties between SAN and ABS Copolymer Matrices Blended with PTFE Polymer Particle Composites

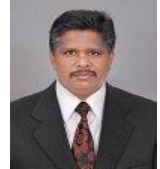

N. Rajini,is currently Professor in Department of Mechanical Engineering, Dean (R \& D) and (Academic), Kalasalingam Academy of Research and Education, Krishnankoil, Tamilnadu, India. He received his Ph.D. degree in Kalasalingam Academy of Research and Education, Krishnankoil, India. His main area of research interest is Polymer nanocomposites. 